\title{
Carleman Estimate for Stochastic Parabolic Equations and Inverse Stochastic Parabolic Problems*
}

\author{
Qi L $\ddot{u}^{\dagger}$
}

\begin{abstract}
In this paper, we establish a global Carleman estimate for stochastic parabolic equations. Based on this estimate, we study two inverse problems for stochastic parabolic equations. One is concerned with a determination problem of the history of a stochastic heat process through the observation at the final time $T$, for which we obtain a conditional stability estimate. The other is an inverse source problem with observation on the lateral boundary. We derive the uniqueness of the source.
\end{abstract}

2010 Mathematics Subject Classification. Primary 65N21, 60H15.

Key Words. Stochastic parabolic equations, Carleman estimate, conditional stability, inverse source problem

\section{Introduction}

In this paper, we study two different inverse problems for stochastic parabolic equations by establishing a global Carleman estimate. We first introduce some notations.

Let $T>0, G \subset \mathbb{R}^{n}(n \in \mathbb{N})$ be a given bounded domain with a $C^{2}$ boundary $\Gamma$. Put

$$
Q \triangleq(0, T) \times G, \quad \Sigma \triangleq(0, T) \times \Gamma .
$$

Let $\left(\Omega, \mathcal{F},\left\{\mathcal{F}_{t}\right\}_{t \geq 0}, P\right)$ be a complete filtered probability space on which a one dimensional standard Brownian motion $\{B(t)\}_{t \geq 0}$ is defined. Let $H$ be a Banach space. Denote by $L_{\mathcal{F}}^{2}(0, T ; H)$ the Banach space consisting of all $H$-valued $\left\{\mathcal{F}_{t}\right\}_{t \geq 0}$-adapted processes $X(\cdot)$ such that $\mathbb{E}\left(|X(\cdot)|_{L^{2}(0, T ; H)}^{2}\right)<\infty$, with the canonical norm; by $L_{\mathcal{F}}^{\infty}(0, T ; H)$ the Banach space consisting of all $H$-valued $\left\{\mathcal{F}_{t}\right\}_{t \geq 0}$-adapted bounded processes; by $L_{\mathcal{F}}^{2}(\Omega ; C([0, T] ; H))$

*This work was partially supported by the NSF of China under grants 11101070, and Grant MTM200803541 of the MICINN, Spain, Project PI2010-04 of the Basque Government, the ERC Advanced Grant FP7-246775 NUMERIWAVES and the ESF Research Networking Programme OPTPDE.

${ }^{\dagger}$ Basque Center for Applied Mathematics (BCAM), Mazarredo 14, 48009, Bilbao, Basque Country, Spain; and School of Mathematical Sciences, University of Electronic Science and Technology of China, Chengdu 610054, China.E-mail: Iuqi59@163.com. 
the Banach space consisting of all $H$-valued $\left\{\mathcal{F}_{t}\right\}_{t \geq 0}$-adapted processes $X(\cdot)$ satisfying that $\mathbb{E}\left(|X(\cdot)|_{C(0, T ; H)}^{2}\right)<\infty$, with the canonical norm(similarly, one can define $L_{\mathcal{F}}^{2}\left(\Omega ; C^{k}([0, T] ; H)\right)$ for any positive $k$ ).

Throughout this paper, we make the following assumptions on the coefficients

$$
b^{i j}: \Omega \times Q \rightarrow \mathbb{R}, \quad(i, j=1,2, \cdots, n):
$$

(H1) $b^{i j} \in L_{\mathcal{F}}^{2}\left(\Omega ; C^{1}\left([0, T] ; W^{2, \infty}(G)\right)\right)$ and $b^{i j}=b^{j i}$;

(H2) There is a constant $\sigma>0$ such that

$$
\sum_{i, j=1}^{n} b^{i j}(\omega, t, x) \xi^{i} \xi^{j} \geq \sigma|\xi|^{2}, \quad(\omega, t, x, \xi) \equiv\left(\omega, t, x, \xi^{1}, \cdots, \xi^{n}\right) \in \Omega \times Q \times \mathbb{R}^{n} .
$$

Let

$$
\begin{gathered}
a_{1} \in L_{\mathcal{F}}^{\infty}\left(0, T ; L^{\infty}\left(G ; \mathbb{R}^{n}\right)\right), \quad a_{2} \in L_{\mathcal{F}}^{\infty}\left(0, T ; L^{\infty}(G)\right) \quad a_{3} \in L_{\mathcal{F}}^{\infty}\left(0, T ; W^{1, \infty}(G)\right), \\
f \in L_{\mathcal{F}}^{2}\left(0, T ; L^{2}(G)\right) \quad \text { and } g \in L_{\mathcal{F}}^{2}\left(0, T ; H^{1}(G)\right)
\end{gathered}
$$

Consider the following stochastic parabolic equation:

$$
\begin{cases}d y-\sum_{i, j=1}^{n}\left(b^{i j} y_{x_{i}}\right)_{x_{j}} d t=\left[\left(a_{1}, \nabla y\right)+a_{2} y+f\right] d t+\left(a_{3} y+g\right) d B & \text { in } Q \\ y=0 & \text { on } \Sigma, \\ y(0)=y_{0}, & \text { in } \Omega\end{cases}
$$

where $y_{0} \in L^{2}\left(\Omega, \mathcal{F}_{0}, P ; L^{2}(G)\right)$ and $y_{x_{i}}=\frac{\partial y}{\partial x_{i}}$.

We first recall the definition of the weak and strong solution of equation (1.2) and give some well-posedness results.

Definition 1.1 We call a stochastic process $y \in L_{\mathcal{F}}^{2}\left(\Omega ; C\left([0, T] ; L^{2}(G)\right)\right) \cap L_{\mathcal{F}}^{2}\left(0, T ; H_{0}^{1}(G)\right) \cap$ $L_{\mathcal{F}}^{2}\left(\Omega ; C\left((0, T] ; H_{0}^{1}(G)\right)\right)$ a weak solution of equation $(1.2)$ if for any $t \in[0, T]$ and any $p \in H_{0}^{1}(G)$, it holds that

$$
\begin{aligned}
& \quad \int_{G} y(t, x) p(x) d x-\int_{G} y_{0}(x) p(x) d x \\
& =\int_{0}^{t} \int_{G}\left\{-\sum_{i, j=1}^{n} b^{i j}(s, x) y_{x_{i}}(s, x) p_{x_{j}}(x)\right. \\
& \left.\quad+\left[\left(a_{1}(s, x), \nabla y(s, x)\right)+a_{2}(s, x) y(s, x)+f(s, x)\right] p(x)\right\} d x d s \\
& +\int_{0}^{t} \int_{G}\left[a_{3}(s, x) y(s, x)+g(s, x)\right] p(x) d x d B, \quad \text { P-a.s. }
\end{aligned}
$$


Definition 1.2 A process $y \in L_{\mathcal{F}}^{2}\left(\Omega ; C\left([0, T] ; H^{2}(G) \cap H_{0}^{1}(G)\right)\right)$ is said to be a strong solution of equation (1.2) if for any $t \in[0, T]$, it holds that

$$
\begin{aligned}
y(t)= & y_{0}+\int_{0}^{t}\left\{-\sum_{i, j=1}^{n}\left(b^{i, j}(s) y_{i}(s)\right)_{j}+\left[\left(a_{1}(s), \nabla y(s)\right)+a_{2}(s) y(s)+f(s)\right]\right\} d s \\
& +\int_{0}^{t} \int_{G}\left[a_{3}(s) y(s)+g(s)\right] d B, \quad \text { P-a.s. }
\end{aligned}
$$

Obviously, strong solution of equation (1.2) is also its weak solution. We have the following well-posedness results for equation (1.2), whose proof can be found in [11, Chapter 6].

Lemma 1.1 There exists a unique weak solution of equation (1.2). Furthermore, it holds that

$$
|y|_{L_{\mathcal{F}}^{2}\left(\Omega ; C\left([0, T] ; L^{2}(G)\right)\right)}+|y|_{L_{\mathcal{F}}^{2}\left(0, T ; H_{0}^{1}(G)\right)} \leq C r_{1}\left|y_{0}\right|_{L^{2}\left(\Omega, \mathcal{F}_{0}, P ; L^{2}(G)\right)} .
$$

Here and in the sequel,

$$
r_{1} \triangleq\left|a_{1}\right|_{L_{\mathcal{F}}^{\infty}\left(0, T ; L^{\infty}\left(G ; \mathbb{R}^{n}\right)\right)}^{2}+\left|a_{2}\right|_{L_{\mathcal{F}}^{\infty}\left(0, T ; L^{\infty}(G)\right)}^{2}+\left|a_{3}\right|_{L_{\mathcal{F}}^{\infty}\left(0, T ; W^{1, \infty}(G)\right)}^{2}+1 .
$$

Lemma 1.2 Let $y_{0} \in L^{2}\left(\Omega, \mathcal{F}_{0}, P ; H^{2}(G) \cap H_{0}^{1}(G)\right), b_{1} \in L_{\mathcal{F}}^{\infty}\left(0, T ; W^{1, \infty}\left(G ; \mathbb{R}^{n}\right)\right), b_{2} \in$ $L_{\mathcal{F}}^{\infty}\left(0, T ; W^{1, \infty}(G)\right)$, and $b_{3} \in L_{\mathcal{F}}^{\infty}\left(0, T ; W^{2, \infty}(G)\right)$. Then there exists a unique strong solution of equation (1.2).

Next, we recall the following Itô's formula, which plays a key role in the sequel.

Lemma 1.3 [Itô's formula] Let $X(\cdot) \in L_{\mathcal{F}}^{2}\left(0, T ; H_{0}^{1}(G)\right)$ be a continuous process with values in $H^{-1}(G)$. Suppose that there exist $X_{0} \in L^{2}\left(\Omega, \mathcal{F}_{0}, P ; L^{2}(G)\right), \Phi(\cdot) \in L_{\mathcal{F}}^{2}\left(0, T ; H^{-1}(G)\right)$ and $\Psi(\cdot) \in L_{\mathcal{F}}^{2}\left(0, T ; L^{2}(G)\right)$ such that for any $t \in[0, T]$, it holds that

$$
X(t)=X_{0}+\int_{0}^{t} \Phi(s) d s+\int_{0}^{t} \Psi(s) d B, P-a . s .
$$

in $H^{-1}(G)$. Then we have that

$$
\begin{aligned}
|X(t)|_{L^{2}(G)}^{2}= & |X(0)|_{L^{2}(G)}^{2}+2 \int_{0}^{t}(X(s), \Phi(s))_{H_{0}^{1}(G), H^{-1}(G)} d s \\
& +2 \int_{0}^{t}(X(s), \Psi(s))_{L^{2}(G)} d B+\int_{0}^{t}|\Psi(s)|_{L^{2}(G)}^{2} d s
\end{aligned}
$$

for arbitrary $t \in[0, T]$.

Remark 1.1 Here we only present a special case for the Itô's formula. It is enough for the proof in our paper. The general form can be found in [25, Chapter 1]. 
Remark 1.2 Obviously, both the weak and strong solution of equation (1.2) satisfy the assumptions for Lemma 1.3. In this paper, we sometimes use the differential form the the above Itô's formula, that is, $d\left(X^{2}\right)=2 X d X+(d X)^{2}$, for the simplicity of notations.

In this paper, we establish a Carleman estimate for equation (1.2). The so-called Carleman estimate is a class of weighted energy estimates which is in connection with (stochastic) differential operators. As far as we know, the first example of such kind of estimate appeared in Carleman's pioneer work for the uniqueness of the solution of first order elliptic system with two variables(see [8]). The idea was generalized to get the uniqueness of the solutions for general Cauchy problems in [6]. Now it is a useful tool for studying the uniqueness and unique continuation property for partial differential equations(see [15] for example). Such kind of estimate has been introduced to solving inverse problems in [4], and were comprehensively studied in [18, 22]. Now it is a helpful methodology for solving inverse problems (e.g. $[18,21,22,29,30])$. Although the form of Carleman estimate seems to be very complex, the idea behind them is very simple. One can understand it by the following example.

Let

$$
\left\{\begin{array}{l}
\frac{d x}{d t}=a(t) x \quad \text { in }(0, T], \\
x(0)=x_{0} .
\end{array}\right.
$$

Here $x_{0} \in \mathbb{R}$ and $a(\cdot) \in L^{\infty}(0, T)$. We prove that there exists a constant $C>0$ such that for any $x_{0} \in \mathbb{R},|x(T)| \leq C\left|x_{0}\right|$ by Carleman estimate. This result is almost trivial. And one can prove it without utilizing Carleman estimate. However, the proof employed here shows all the ideas of Carleman estimate.

Let $\tilde{x}(t)=e^{-\varsigma t} x(t)$ with $\varsigma \geq 0$. Then we have

$$
\frac{d \tilde{x}^{2}}{d t}=2 a(t) \tilde{x}^{2}-2 \varsigma \tilde{x}^{2}=2[a(t)-\varsigma] \tilde{x}^{2} .
$$

If we choose $\varsigma \geq|a|_{L^{\infty}(0, T)}$, then we know that $\frac{d \tilde{x}^{2}}{d t} \leq 0$, which implies that $\tilde{x}^{2}(T) \leq \tilde{x}^{2}(0)$. Hence, we get

$$
e^{-2 \varsigma T} x^{2}(T) \leq x^{2}(0) .
$$

From this, we obtain $|x(T)| \leq e^{T|a|_{L} \infty(0, T)}\left|x_{0}\right|$ immediately. Thus, we prove the desired result and we know $C$ can be chosen to be $e^{T|a|_{L} \infty(0, T)}$.

Inequality (1.9) is a kind of Carleman estiamte. The function $e^{-\varsigma t}$ is called weight function and $\varsigma$ is a parameter which can be chosen for our purpose. By means of the choice of $\varsigma$, we control the lower order term $a(t) x$ and obtain inequality (1.9). For (stochastic) partial differential equations, both the choice of the weight function and the computation are much more complex. However, they enjoy the same idea.

Now we introduce the Carleman estimate to be established in this paper. To start with, we give some functions. Let $s \in(0,+\infty), t \in(0,+\infty)$, and $\psi \in C^{\infty}(\mathbb{R})$ with $\left|\psi_{t}\right| \geq 1$, which is independent of the $x$-variable. Put

$$
\varphi=e^{\lambda \psi} \text { and } \theta=e^{s \varphi} \text {. }
$$

We have the following result. 
Theorem 1.1 Let $\delta \in[0, T)$. Let $\varphi$ and $\theta$ be given in (1.10). There exists a $\lambda_{1}>0$ such that for all $\lambda \geq \lambda_{1}$, there exists an $s_{0}\left(\lambda_{1}\right)>0$ so that for all $s \geq s_{0}\left(\lambda_{1}\right)$, it holds that

$$
\begin{aligned}
& \lambda \mathbb{E} \int_{\delta}^{T} \int_{G} \theta^{2}|\nabla y|^{2} d x d t+s \lambda^{2} \mathbb{E} \int_{\delta}^{T} \int_{G} \varphi \theta^{2} y^{2} d x d t \\
\leq & C \mathbb{E}\left[\theta^{2}(T)|\nabla y(T)|_{L^{2}(G)}^{2}+\theta^{2}(\delta)|\nabla y(\delta)|_{L^{2}(G)}^{2}+s \lambda \varphi(T) \theta^{2}(T)|y(T)|_{L^{2}(G)}^{2}\right. \\
& \left.+s \lambda \varphi(\delta) \theta^{2}(\delta)|y(\delta)|_{L^{2}(G)}^{2}+\int_{\delta}^{T} \int_{G}(1+\varphi) \theta^{2}\left(f^{2}+g^{2}+|\nabla g|^{2}\right) d x d t\right],
\end{aligned}
$$

Here $y$ is arbitrary weak solution of equation (1.2).

Here and in the sequel, the constant $C$ depends only on $G,\left(b^{i j}\right)_{n \times n}, T, \delta$ and $\psi$, which may change from line to line.

Although there are numerous results for the global Carleman estimate for deterministic parabolic equations(see [13, 29] for example), people know very little about the stochastic counterpart. In fact, as far as we know, $[2,28]$ are the only two published papers addressing the global Carleman estimate for stochastic parabolic equations. In [2, 28], some Carlemantype inequalities were established, for deriving the null controllability of stochastic parabolic equations. Note further that the weight function $\theta$ used in this paper (which plays a key role in the sequel) is quite different from that in $[2,28]$. It seems that the Carleman estimate in $[2,28]$ cannot be applied to studying the inverse problems introduced in ths sequel. Indeed, the weight function $\theta$ in $[2,28]$ is supposed to vanish at 0 and $T$, and therefore it does not serve the purpose of proving Theorem 1.2 and Theorem 1.4.

As applications of Theorem 1.1, we study two inverse problems for stochastic parabolic equations. There are abundant works addressing the inverse problems for PDEs. And it is even impossible to list the related papers owing to the big amount. However, there exist a very few works addressing inverse problems for stochastic PDEs (see [3, 9, 16] for example). Although there are some people considering the inverse source problem for parabolic equations with random noise in the measurement (see [20] for example), to the best of our knowledge, there is no paper considering the inverse problem for stochastic parabolic equations.

Now we introduce the inverse problems studied in this paper. Consider the following stochastic parabolic equation:

$$
\begin{cases}d y-\sum_{i, j=1}^{n}\left(b^{i j} y_{x_{i}}\right)_{x_{j}} d t=\left[\left(a_{1}, \nabla y\right)+a_{2} y\right] d t+a_{3} y d B & \text { in } Q, \\ y=0 & \text { on } \Sigma, \\ y(0)=y_{0} & \text { in } \Omega .\end{cases}
$$

Here $y_{0} \in L^{2}\left(\Omega, \mathcal{F}_{0}, P\right)$.

The first inverse problem is concerned with the following problem:

Stochastic parabolic equation backward in time: Let $0 \leq t_{0}<T$. Determine $y\left(\cdot, t_{0}\right)$, P-a.s. from $y(\cdot, T)$. 
For deterministic parabolic equations, such kind of problem has lots of applications in mathematical physics (e.g. [1]) and is studied extensively (see [30] for a nice survey). Generally speaking, the problem of (stochastic) parabolic equation backward in time is ill-posed. Small errors in the measuring of the terminal data may cause huge deviations in final results, that is, there is no stability in this problem. Fortunately, if we assume a priori bound for $y(0)$ (such assumption is reasonable from a practical viewpoint), then we can regain the stability in some sense. The concept of conditional stability is used to describe such kind of stability. In general framework, the conditional stability problem can be formulated as follows:

$$
\begin{aligned}
& \text { Let } t_{0} \in[0, T), \alpha_{1} \geq 0, \alpha_{2} \geq 0 \text { and } M>0 \text {. Put } \\
& \qquad U_{M, \alpha_{1}} \triangleq\left\{f \in L^{2}\left(\Omega, P, \mathcal{F}_{0} ; H^{\alpha_{1}}(G)\right):|f|_{L^{2}\left(\Omega, P, \mathcal{F}_{0} ; H^{\alpha_{1}}(G)\right)} \leq M\right\} .
\end{aligned}
$$

If $y_{0} \in U_{M, \alpha_{1}}$, then can we choose a function $\beta \in C[0,+\infty)$ satisfying the following properties:

$$
\left\{\begin{array}{l}
\text { 1. } \beta \geq 0 \text { and } \beta \text { is strictly increasing; } \\
\text { 2. } \lim _{\eta \rightarrow 0} \beta(\eta)=0 ; \\
\text { 3. }\left|y\left(t_{0}\right)\right|_{L^{2}\left(\Omega, \mathcal{F}_{t_{0}}, P ; H^{\alpha_{1}}(G)\right)} \leq \beta\left(|y(T)|_{L^{2}\left(\Omega, \mathcal{F}_{T}, P ; H^{\alpha}(G)\right)}\right) .
\end{array}\right.
$$

Remark 1.3 Here we expect the existence of $\beta$ with the assumptions that $y_{0}$ belongs to a special set $U_{M, \alpha_{1}}$, which means that $y_{0}$ enjoys a priori bound in some sense. Generally speaking, $\beta$ depends on $M$ and $\alpha_{1}$. Once we choose $M$ and $\alpha_{1}$, we add some conditions to the initial data of equation (1.12). Hence, that the stability result implied by $\beta$ depends on our choice of the initial data. This is why we call it "conditional stability".

Remark 1.4 The first property for $\beta$ means that we only choose $\beta$ in a special class of functions, that is, the strictly increasing functions. The second and the third property guarantee the conditional stability. Without assuming property 2, we can always construct $\beta$ as $\beta(x)=C+x$ with a constant $C$ which is large enough. However, such kind of functions do not make any sense for conditional stability.

Remark 1.5 Once $\beta$ exists, it is not unique. For example, $\tilde{b}(x)=\beta(x)+x$ is another function satisfying the three properties.

In this paper, we obtain the following interpolation inequality for the weak solution of equation (1.12), which implies a conditional stability result for equation (1.12) backward in time.

Theorem 1.2 Let $t_{0} \in[0, T]$. Then there exist a constant $\theta \in(0,1)$ and a constant $C>0$ such that

$$
\left|y\left(t_{0}\right)\right|_{L^{2}\left(\Omega, \mathcal{F}_{t_{0}}, P ; L^{2}(G)\right)} \leq C|y|_{L_{\mathcal{F}}^{2}\left(0, T ; L^{2}(G)\right)}^{1-\theta}|y(T)|_{L^{2}\left(\Omega, \mathcal{F}_{T}, P ; H^{1}(G)\right)}^{\theta},
$$

for any $y$ solving equation (1.12) in the sense of weak solution. 
As a consequence, we obtain the following result.

Theorem 1.3 Let $y_{0} \in U_{M, 0}, \alpha_{2}=1$ and $\beta(x)=C M^{1-\theta} x^{\theta}$ with a constant $C$ independent of $y(0)$. Then we have

$$
\left|y\left(t_{0}\right)\right|_{L^{2}\left(\Omega, \mathcal{F}_{t_{0}}, P ; L^{2}(G)\right)} \leq \beta\left(|y(T)|_{L^{2}\left(\Omega, \mathcal{F}_{T}, P ; H^{1}(G)\right)}\right) .
$$

The proof of Theorem 1.3 follows Lemma 1.1 and Theorem 1.2 immediately. We omit it here.

In deterministic setting, a result which is stronger than Theorem 1.2 was obtained in [26], where the authors study the following equation:

$$
\begin{cases}y_{t}-\Delta y=b y & \text { in } Q \\ y=0 & \text { on } \Sigma .\end{cases}
$$

Here $b$ is a suitable function. With the assumption that $G$ is convex, they get

$$
|y(0)|_{L^{2}(G)}^{2} \leq C \exp \left(\frac{|y(0)|_{L^{2}(G)}}{|y(0)|_{H^{-1}(G)}}\right)|y(T)|_{L^{2}\left(G_{0}\right)}^{2} .
$$

Here $G_{0}$ is any open subset of $G$. Compared with Theorem 1.2, only $|y(T)|_{L^{2}\left(G_{0}\right)}^{2}$ is involved in the right hand side of the inequality. They prove this result by employing some special frequency functions, which were first constructed for proving the doubling property of the solution of heat equations. However, since the solution of equation (1.12) is non-differentiable with respect to $t$, it seems that their method cannot be easily adopted to solve our problem.

As another consequence of Theorem 1.2, we get a backward uniqueness for equation (1.12).

Corollary 1.1 Assume that $y$ is a weak solution of equation (1.12). If $y(T)=0$ in $G$, $P$-a.s., then $y(t)=0$ in $G, P$-a.s. for all $t \in[0, T]$.

The uniqueness problems for the solutions of both deterministic and stochastic partial differential equations have been studied for a long time. There are a great many positive results and some negative results. In case of time reversible systems, the backward uniqueness is equivalent to the classical (forward) uniqueness. If one considers time irreversible systems, such as parabolic equations, the situation is quite different. The backward uniqueness implies the classical (forward) uniqueness, however, generally speaking, the converse conclusion is untrue.

On account of the plentiful applications, such as studying the long time behavior of solutions and establishing the approximate controllability from the null controllability, the backward uniqueness for parabolic equations draws lots of attention(see [12, 14, 23, 24, 27] and the references cited therein). It is well understood now. On the contrast, as far as we know, [5] is the only paper concerned with backward uniqueness for stochastic parabolic equations in the literature. In [5], the authors obtained the backward uniqueness for semilinear stochastic parabolic equations with deterministic coefficients. They employed some deep 
tools in Stochastic Analysis to establish the result. However, it seems that their method depends on the very fact that the coefficients are deterministic and one cannot simply mimic their method to obtain Corollary 1.1, since the coefficients are random.

The other inverse problem studied in this paper is about the global uniqueness of an inverse source problem for stochastic parabolic equations. We first give a precise formulation of the problem.

Let $x=\left(x_{1}, x^{\prime}\right) \in \mathbb{R}^{n}$ and $x^{\prime}=\left(x_{2}, \cdots, x_{n}\right) \in \mathbb{R}^{n-1}$. Consider a special $G$ as $G=$ $(0, l) \times G^{\prime}$, where $G^{\prime} \subset \mathbb{R}^{n-1}$ be a bounded domain with a $C^{2}$ boundary. We consider the following stochastic parabolic equation:

$$
\begin{cases}d y-\Delta y=\left[\left(b_{1}, \nabla y\right)+b_{2} y+h\left(t, x^{\prime}\right) R(t, x)\right] d t+b_{3} y d B(t) & \text { in } Q \\ y=0 & \text { on } \Sigma \\ y(0)=0 & \text { in } G\end{cases}
$$

Here

$$
b_{1} \in L_{\mathcal{F}}^{\infty}\left(0, T ; W^{1, \infty}\left(G ; \mathbb{R}^{n}\right)\right), \quad b_{2} \in L_{\mathcal{F}}^{\infty}\left(0, T ; W^{1, \infty}(G)\right), \quad b_{3} \in L_{\mathcal{F}}^{\infty}\left(0, T ; W^{2, \infty}(G)\right),
$$

and

$$
R \in C^{2}([0, T] \times \bar{G}), \quad h \in L_{\mathcal{F}}^{2}\left(0, T ; H^{1}\left(G^{\prime}\right)\right) .
$$

The inverse source problem studied here is as follows:

Let $R$ be given and $0<t_{0}<T$. Determine the source function $h\left(t, x^{\prime}\right),\left(t, x^{\prime}\right) \in\left(0, t_{0}\right) \times$ $G^{\prime}$, by means of the observation of $\left.\frac{\partial y}{\partial \nu}\right|_{\left[0, t_{0}\right] \times \partial G}$.

Here $\nu=\left(\nu^{1}, \cdots, \nu^{n}\right) \in \mathbb{R}^{n}$ is the outer normal vector of $\Gamma$.

We have the following uniqueness result about the above problem.

Theorem 1.4 Let

$$
|R(t, x)| \neq 0 \text { for all }(t, x) \in\left[0, t_{0}\right] \times \bar{G}
$$

If

$$
\frac{\partial y}{\partial \nu}=0 \text { on }\left[0, t_{0}\right] \times \partial G, P-a . s .
$$

then

$$
h\left(t, x^{\prime}\right)=0 \text { for all }\left(t, x^{\prime}\right) \in\left[0, t_{0}\right] \times G^{\prime}, P \text {-a.s. }
$$

Remark 1.6 One can follow the proof of Theorem 1.4 to show that Theorem 1.4 also holds when $\Delta y$ is substituted by $\sum_{i, j=1}^{n}\left(b^{i j} y_{i}\right)_{j}$. Here we consider equation (1.16) for the sake of presenting the key idea in a simple way. 
In practical problems, it is important to specify some proper data so that the parameter to be reconstructed is uniquely identifiable. In our model, the data utilized is the boundary normal derivative of the solution. This type of inverse problem is important in many branches of engineering sciences. For examples, an accurate estimation of a pollution source in a river, a determination of magnitude of groundwater pollution sources.

In the literature, determining a spacewise dependent source function for parabolic equations has been considered comprehensively(see [7, 10, 18, 19, 30] and the references cited therein). A classical result for the deterministic setting is as follows.

Consider the following parabolic equation:

$$
\begin{cases}y_{t}-\Delta y=c_{1} \nabla y+c_{2} y+R f & \text { in } Q \\ y=0 & \text { on } \Sigma .\end{cases}
$$

Here $c_{1}$ and $c_{2}$ are suitable functions on $Q . R \in L^{\infty}(Q), R_{t} \in L^{\infty}(Q)$ and $R\left(t_{0}, x\right) \neq 0$ in $\bar{G}$ for some $t_{0} \in(0, T] . f \in L^{2}(G)$ is independent of $t$. The authors in [17] proved the following result:

Assume that $y \in H^{2,1}(Q)$ and $y_{t} \in H^{2,1}(Q)$, then there exists a constant $C>0$ such that

$$
|f|_{L^{2}(G)} \leq C\left(\left|y\left(t_{0}\right)\right|_{H^{2}(G)}+\left|\frac{\partial y_{t}}{\partial \nu}\right|_{L^{2}\left(0, T ; L^{2}\left(\Gamma_{0}\right)\right)}\right),
$$

where $\Gamma_{0}$ is any open subset of $\Gamma$.

Compared with Theorem 1.4, inequality (1.19) gives an explicit estimate for the source term by $\left|y\left(t_{0}\right)\right|_{H^{2}(G)}$ and $\left|\frac{\partial y_{t}}{\partial \nu}\right|_{L^{2}\left(0, T ; L^{2}\left(\Gamma_{0}\right)\right)}$. A key step in the proof of equality (1.19) is to differentiate the solution of (1.18) with respect to $t$. Unfortunately, the solution of (1.16) does not enjoy differentiability with respect to $t$ since the effect of the stochastic noise. However, we can borrow some idea from the proof of inequality (1.19). Although it is impossible for us to assume that the solution of equation (1.16) is differentiable with respect to $t$, we can show that it is differentiable with respect to $x$ with some assumptions(the assumptions in this paper is enough). In this case, we can show the uniqueness of $h$ if $h$ is independent of some $x_{i}(i=1, \cdots, n)$. Here we suppose that $h$ is independent of $x_{1}$.

Obviously, both equation (1.12) and equation (1.16) are special examples of equation (1.2).

Remark 1.7 As we have pointed out, the non-differentiability with respect to the variable with noise (say, the time variable considered in this paper) of the solution of a stochastic PDE usually leads to substantially new difficulties in the study of inverse problems for stochastic PDEs. Another trouble for studying the inverse problem of stochastic PDEs is that the usual compactness embedding result does not remain true for the solution spaces related to stochastic PDEs. Due to these new difficulties, some useful methods for solving inverse problems for deterministic PDEs (see [18, 22] for example) cannot be used to solve the corresponding inverse problems in the stochastic setting.

The rest paper is organized as follows. In Section 2, we prove Theorem 1.1. Section 3 is addressed the proof of Theorem 1.2. At last, in Section 4, we give a proof for Theorem 1.4. 


\section{Carleman estimate for stochastic parabolic equations}

In this section, we prove Theorem 1.1.

We first give a weighted identity, which plays an important role in the proof of Theorem 1.1.

Proposition 2.1 Assume that $u$ is an $H^{2}\left(\mathbb{R}^{n}\right)$-valued continuous semi-martingale. Put $v=$ $\theta u($ recall (1.10) for the definition of $\theta)$. Then we have the following equality:

$$
\begin{aligned}
& -\theta\left[\sum_{i, j=1}^{n}\left(b^{i j} v_{x_{i}}\right)_{x_{j}}+s \lambda \varphi \psi_{t} v\right]\left[d u-\sum_{i, j=1}^{n}\left(b^{i j} u_{x_{i}}\right)_{x_{j}} d t\right]+\frac{1}{4} \lambda \theta v\left[d u-\sum_{i, j=1}^{n}\left(b^{i j} u_{x_{i}}\right)_{x_{j}} d t\right] \\
= & -\sum_{i, j=1}^{n}\left(b^{i j} v_{x_{i}} d v+\frac{1}{4} b^{i j} v_{x_{i}} v d t\right)_{x_{j}}+\frac{1}{2} d\left(\sum_{i, j=1}^{n} b^{i j} v_{x_{i}} v_{x_{j}}-s \lambda \varphi \psi_{t} v^{2}+\frac{1}{8} \lambda v^{2}\right) \\
& -\left(\frac{1}{2} \sum_{i, j=1}^{n} b^{i j} d v_{x_{i}} d v_{x_{j}}+\frac{1}{2} \sum_{i, j=1}^{n} b_{t}^{i j} v_{x_{i}} v_{x_{j}} d t-\frac{1}{4} \lambda \sum_{i, j=1}^{n} b^{i j} v_{x_{i}} v_{x_{j}} d t\right) \\
& +\frac{1}{2} s \lambda^{2} \varphi \psi_{t}^{2} v^{2} d t+\frac{1}{2} s \lambda \varphi \psi_{t t} v^{2} d t-\frac{1}{4} s \lambda^{2} \psi_{t} \varphi v^{2} d t+\frac{1}{2} s \lambda \varphi \psi_{t}(d v)^{2}-\frac{1}{8} \lambda(d v)^{2} \\
& +\left[\sum_{i, j=1}^{n}\left(b^{i j} v_{x_{i}}\right)_{x_{j}}+s \lambda \varphi \psi_{t} v\right]^{2} d t .
\end{aligned}
$$

Proof: The proof is based on some direct computation by Itô's stochastic calculus. The first term in the left hand side of equality (2.1) reads as

$$
\begin{aligned}
& -\theta\left[\sum_{i, j=1}^{n}\left(b^{i j} v_{x_{i}}\right)_{x_{j}}+s \lambda \varphi \psi_{t} v\right]\left[d u-\sum_{i, j=1}^{n}\left(b^{i j} u_{x_{i}}\right)_{x_{j}} d t\right] \\
= & -\left[\sum_{i, j=1}^{n}\left(b^{i j} v_{x_{i}}\right)_{x_{j}}+s \lambda \varphi \psi_{t} v\right]\left[d v-\sum_{i, j=1}^{n}\left(b^{i j} v_{x_{i}}\right)_{x_{j}} d t-s \lambda \varphi \psi_{t} v d t\right] \\
= & -\sum_{i, j=1}^{n}\left(b^{i j} v_{x_{i}}\right)_{x_{j}} d v-s \lambda \varphi \psi_{t} v d v+\left[\sum_{i, j=1}^{n}\left(b^{i j} v_{x_{i}}\right)_{x_{j}}+s \lambda \varphi \psi_{t} v\right]^{2} d t \\
= & -\sum_{i, j=1}^{n}\left(b^{i j} v_{x_{i}} d v\right)_{x_{j}}+\frac{1}{2} \sum_{i, j=1}^{n} d\left(b^{i j} v_{x_{i}} v_{x_{j}}\right)-\frac{1}{2} \sum_{i, j=1}^{n} b^{i j} d v_{x_{i}} d v_{x_{j}} \\
& -\frac{1}{2} \sum_{i, j=1}^{n} b_{t}^{i j} v_{x_{i}} v_{x_{j}} d t-\frac{1}{2} d\left(s \lambda \varphi \psi_{t} v^{2}\right)+\frac{1}{2} s \lambda^{2} \psi_{t}^{2} \varphi v^{2} d t \\
& +\frac{1}{2} s \lambda \varphi \psi_{t t} v^{2} d t+\frac{1}{2} s \lambda \psi_{t} \varphi(d v)^{2}+\left[\sum_{i, j=1}^{n}\left(b^{i j} v_{x_{i}}\right)_{x_{j}}+s \lambda \varphi \psi_{t} v\right]^{2} d t .
\end{aligned}
$$


The second term in the left hand side of equality (2.1) satisfies

$$
\begin{aligned}
& \frac{1}{4} \lambda \theta v\left[d u-\sum_{i, j=1}^{n}\left(b^{i j} u_{x_{i}}\right)_{x_{j}} d t\right] \\
= & \frac{1}{4} \lambda v\left[d v-\sum_{i, j=1}^{n}\left(b^{i j} v_{x_{i}}\right)_{x_{j}} d t-s \lambda \varphi \psi_{t} v d t\right] \\
= & \frac{1}{4} \lambda v d v-\frac{1}{4} \lambda v \sum_{i, j=1}^{n}\left(b^{i j} v_{x_{i}}\right)_{x_{j}} d t-\frac{1}{4} s \lambda^{2} \varphi \psi_{t} v^{2} d t \\
= & \frac{1}{8} \lambda d v^{2}-\frac{1}{8} \lambda(d v)^{2}-\frac{1}{4} \lambda \sum_{i, j=1}^{n}\left(b^{i j} v_{x_{i}} v\right)_{x_{j}} d t+\frac{1}{4} \lambda \sum_{i, j=1}^{n} b^{i j} v_{x_{i}} v_{x_{j}} d t-\frac{1}{4} s \lambda^{2} \psi_{t} \varphi v^{2} d t .
\end{aligned}
$$

This, together with equality (2.2), implies equality (2.1).

Now we are in a position to prove Theorem 1.1.

Proof of Theorem 1.1: Applying Proposition 2.1 to equation (1.2) with $u=y$, integrating equality $(2.1)$ on $[\delta, T] \times G$ for some $\delta \in[0, T)$, and taking mathematical expectation, we get that

$$
\begin{aligned}
& -\mathbb{E} \int_{\delta}^{T} \int_{G} \theta\left[\sum_{i, j=1}^{n}\left(b^{i j} v_{x_{i}}\right)_{x_{j}}+s \lambda \varphi \psi_{t} v\right]\left[d u-\sum_{i, j=1}^{n}\left(b^{i j} u_{x_{i}}\right)_{x_{j}} d t\right] d x \\
& +\frac{1}{4} \lambda \mathbb{E} \int_{\delta}^{T} \int_{G} \theta v\left[d y-\sum_{i, j=1}^{n}\left(b^{i j} y_{x_{i}}\right)_{x_{j}} d t\right] d x \\
& =-\mathbb{E} \int_{\delta}^{T} \int_{G} \sum_{i, j=1}^{n}\left(b^{i j} v_{x_{i}} d v+\frac{1}{4} \lambda b^{i j} v_{x_{i}} v d t\right)_{x_{j}} d x+\frac{1}{2} \mathbb{E} \int_{\delta}^{T} \int_{G} d\left(\sum_{i, j=1}^{n} b^{i j} v_{x_{i}} v_{x_{j}}-s \lambda \varphi \psi_{t} v^{2}+\frac{1}{8} \lambda v^{2}\right) d x \\
& -\mathbb{E} \int_{\delta}^{T} \int_{G}\left(\frac{1}{4} \lambda \sum_{i, j=1}^{n} b^{i j} v_{x_{i}} v_{x_{j}} d t+\frac{1}{2} \sum_{i, j=1}^{n} b_{t}^{i j} v_{x_{i}} v_{x_{j}} d t-\frac{1}{2} \sum_{i, j=1}^{n} b^{i j} d v_{x_{i}} d v_{x_{j}}\right) d x \\
& +\mathbb{E} \int_{\delta}^{T} \int_{G}\left[\frac{1}{2} s \lambda^{2} \varphi \psi_{t}^{2} v^{2} d t+\frac{1}{2} s \lambda \varphi \psi_{t t} v^{2} d t-\frac{1}{4} s \lambda^{2} \varphi \psi_{t} v^{2} d t+\frac{1}{2} s \lambda \varphi \psi_{t}(d v)^{2}-\frac{1}{8} \lambda(d v)^{2}\right] d x \\
& +\mathbb{E} \int_{\delta}^{T} \int_{G}\left[\sum_{i, j=1}^{n}\left(b^{i j} v_{x_{i}}\right)_{x_{j}}+s \lambda \varphi \psi_{t} v\right]^{2} d x d t .
\end{aligned}
$$

Now we estimate the terms in the right hand side of equality (2.2) one by one.

For the first one, since $\left.y\right|_{\Sigma}=0$, we have that $\left.v\right|_{\Sigma}=0$. Therefore, it holds that

$$
\begin{aligned}
& -\mathbb{E} \int_{\delta}^{T} \int_{G} \sum_{i, j}^{n}\left(b^{i j} v_{x_{i}} d v+\frac{1}{4} \lambda b^{i j} v_{x_{i}} v d t\right)_{x_{j}} d x \\
= & -\mathbb{E} \int_{\delta}^{T} \int_{\Gamma} \sum_{i, j=1}^{n} b^{i j}\left(v_{x_{i}} d v+\frac{1}{4} \lambda v_{x_{i}} v d t\right) \nu^{j} d \Gamma=0 .
\end{aligned}
$$


For the second one, we have

$$
\begin{aligned}
& \frac{1}{2} \mathbb{E} \int_{\delta}^{T} \int_{G} d\left(\sum_{i, j=1}^{n} b^{i j} v_{x_{i}} v_{x_{j}}-s \lambda \varphi \psi_{t} v^{2}+\frac{1}{8} \lambda v^{2}\right) d x \\
\geq & -C \mathbb{E}\left(|\nabla v(T)|_{L^{2}(G)}^{2}+|\nabla v(\delta)|_{L^{2}(G)}^{2}+s \lambda \varphi(T)|v(T)|_{L^{2}(G)}^{2}+s \lambda \varphi(\delta)|v(\delta)|_{L^{2}(G)}^{2}\right) .
\end{aligned}
$$

Since

$$
\begin{aligned}
& \mathbb{E} \int_{\delta}^{T} \int_{G} \frac{1}{2} \sum_{i, j=1}^{n} b^{i j} d v_{x_{i}} d v_{x_{j}} d x \\
= & \frac{1}{2} \mathbb{E} \int_{\delta}^{T} \int_{G} \sum_{i, j=1}^{n} b^{i j} \theta^{2}\left(a_{3} y+g\right)_{x_{i}}\left(a_{3} y+g\right)_{x_{j}} d x d t,
\end{aligned}
$$

the third one reads as

$$
\begin{aligned}
& \mathbb{E} \int_{\delta}^{T} \int_{G}\left(\frac{1}{4} \lambda \sum_{i, j=1}^{n} b^{i j} v_{x_{i}} v_{x_{j}} d t+\frac{1}{2} \sum_{i, j=1}^{n} b_{t}^{i j} v_{x_{i}} v_{x_{j}} d t-\frac{1}{2} \sum_{i, j=1}^{n} b^{i j} d v_{x_{i}} d v_{x_{j}}\right) d x \\
\geq & \mathbb{E} \int_{\delta}^{T} \int_{G}\left[\frac{1}{4} \lambda \sigma|\nabla v|^{2}-C|\nabla v|^{2}-C\left(a_{3}^{2}|\nabla v|^{2}+\left|\nabla a_{3}\right|^{2} v^{2}+\theta^{2}|\nabla g|^{2}+\theta^{2}|g|^{2}\right)\right] d x d t \\
\geq & \frac{1}{4} \lambda \mathbb{E} \int_{\delta}^{T} \int_{G} \sigma|\nabla v|^{2} d x d t-C\left(\left|a_{3}\right|_{L_{\mathcal{F}}\left(0, T ; W^{1, \infty}(G)\right)}^{2}+1\right) \mathbb{E} \int_{\delta}^{T} \int_{G}\left(|\nabla v|^{2}+v^{2}\right) d x d t \\
& -C \mathbb{E} \int_{\delta}^{T} \int_{G} \theta^{2}\left(|\nabla g|^{2}+g^{2}\right) d x d t .
\end{aligned}
$$

For the forth one, recalling that $\left|\psi_{t}\right| \geq 1$ and utilizing that

$$
\begin{aligned}
& \mathbb{E} \int_{\delta}^{T} \int_{G}\left[\frac{1}{2} s \lambda \varphi \psi_{t}(d v)^{2}-\frac{1}{8} \lambda(d v)^{2}\right] d x \\
= & \mathbb{E} \int_{\delta}^{T} \int_{G} \theta^{2}\left[\frac{1}{2} s \lambda \varphi \psi_{t}\left(a_{3} y+g\right)^{2}-\frac{1}{8} \lambda\left(a_{3} y+g\right)^{2}\right] d x d t,
\end{aligned}
$$

we see

$$
\begin{aligned}
& \mathbb{E} \int_{\delta}^{T} \int_{G}\left[\frac{1}{2} s \lambda^{2} \varphi \psi_{t}^{2} v^{2} d t+\frac{1}{2} s \lambda \varphi \psi_{t t} v^{2} d t-\frac{1}{4} s \lambda^{2} \varphi \psi_{t} v^{2} d t+\frac{1}{2} s \lambda \varphi \psi_{t}(d v)^{2}-\frac{1}{8} \lambda(d v)^{2}\right] d x \\
& \geq \frac{1}{4} s \lambda^{2} \mathbb{E} \int_{\delta}^{T} \int_{G} \varphi v^{2} d x d t+s O(\lambda) \mathbb{E} \int_{\delta}^{T} \int_{G} \varphi v^{2} d x d t-C s \lambda \mathbb{E} \int_{\delta}^{T} \int_{G}(1+\varphi) \theta^{2} g^{2} d x d t .
\end{aligned}
$$

Thus, we know that there exists a $\lambda_{0}>0$ such that for all $\lambda \geq \lambda_{0}$, it holds that

$$
\begin{aligned}
& \mathbb{E} \int_{\delta}^{T} \int_{G}\left[\frac{1}{2} s \lambda^{2} \varphi \psi_{t}^{2} v^{2} d t+\frac{1}{2} s \lambda \varphi \psi_{t t} v^{2} d t-\frac{1}{4} s \lambda^{2} \varphi \psi_{t} v^{2} d t+\frac{1}{2} s \lambda \varphi \psi_{t}(d v)^{2}-\frac{1}{8} \lambda(d v)^{2}\right] d x \\
& \geq \frac{1}{8} s \lambda^{2} \mathbb{E} \int_{\delta}^{T} \int_{G} \varphi v^{2} d x d t-C s \lambda \mathbb{E} \int_{\delta}^{T} \int_{G}(1+\varphi) \theta^{2} g^{2} d x d t .
\end{aligned}
$$


Now, we estimate the terms in the left hand side one by one. By equation (1.2) and noting that

$$
-\mathbb{E} \int_{\delta}^{T} \int_{G} \theta\left[\sum_{i, j=1}^{n}\left(b^{i j} v_{x_{i}}\right)_{x_{j}}+s \lambda \varphi \psi_{t} v\right]\left(a_{3} y+g\right) d B d x=0
$$

we know that

$$
\begin{aligned}
& -\mathbb{E} \int_{\delta}^{T} \int_{G} \theta\left[\sum_{i, j=1}^{n}\left(b^{i j} v_{x_{i}}\right)_{x_{j}}+s \lambda \varphi \psi_{t} v\right]\left[d y-\sum_{i, j=1}^{n}\left(b^{i j} y_{x_{i}}\right)_{x_{j}} d t\right] d x \\
= & -\mathbb{E} \int_{\delta}^{T} \int_{G} \theta\left[\sum_{i, j=1}^{n}\left(b^{i j} v_{x_{i}}\right)_{x_{j}}+s \lambda \varphi \psi_{t} v\right]\left[\left(a_{1}, \nabla y\right)+a_{2} y+f\right] d t d x \\
\leq & \mathbb{E} \int_{\delta}^{T} \int_{G}\left[\sum_{i, j=1}^{n}\left(b^{i j} v_{x_{i}}\right)_{x_{j}}+s \lambda \varphi \psi_{t} v\right]^{2} d x d t+\mathbb{E} \int_{\delta}^{T} \int_{G} \theta^{2}\left[\left(a_{1}, \nabla y\right)+a_{2} y+f\right]^{2} d t d x \\
\leq & \mathbb{E} \int_{\delta}^{T} \int_{G}\left[\sum_{i, j=1}^{n}\left(b^{i j} v_{x_{i}}\right)_{x_{j}}+s \lambda \varphi \psi_{t} v\right]^{2} d x d t+3 \mathbb{E} \int_{\delta}^{T} \int_{G} \theta^{2}\left(\left|a_{1}\right|^{2}|\nabla u|^{2}+a_{2}^{2} u^{2}+f^{2}\right) d x d t \\
\leq & \mathbb{E} \int_{\delta}^{T} \int_{G}\left[\sum_{i, j=1}^{n}\left(b^{i j} v_{x_{i}}\right)_{x_{j}}+s \lambda \varphi \psi_{t} v\right]^{2} d x d t+3\left|a_{1}\right|_{L_{\mathcal{F}}^{\infty}\left(0, T ; L^{\infty}\left(G ; \mathbb{R}^{n}\right)\right)}^{2} \mathbb{E} \int_{\delta}^{T} \int_{G}|\nabla v|^{2} d x d t \\
& +3\left|a_{2}\right|_{L_{\mathcal{F}}^{\infty}\left(0, T ; L^{\infty}(G)\right)}^{2} \mathbb{E} \int_{\delta}^{T} \int_{G} v^{2} d x d t+3 \mathbb{E} \int_{\delta}^{T} \int_{G} \theta^{2} f^{2} d x d t,
\end{aligned}
$$

and that

$$
\begin{aligned}
& \frac{1}{4} \lambda \mathbb{E} \int_{\delta}^{T} \int_{G} \theta v\left[d u-\sum_{i, j=1}^{n}\left(b^{i j} u_{x_{i}}\right)_{x_{j}} d t\right] d x \\
= & \frac{1}{4} \lambda \mathbb{E} \int_{\delta}^{T} \int_{G} \theta v\left[\left(a_{1}, \nabla y\right)+a_{2} y+f\right] d t d x \\
\leq & \frac{1}{64} \lambda \mathbb{E} \int_{\delta}^{T} \int_{G} v^{2} d x d t+\mathbb{E} \int_{\delta}^{T} \int_{G} \theta^{2}\left[\left(a_{1}, \nabla y\right)+a_{2} y+f\right]^{2} d t d x \\
\leq & \frac{1}{64} \lambda^{2} \mathbb{E} \int_{\delta}^{T} \int_{G} v^{2} d x d t+3 \mathbb{E} \int_{\delta}^{T} \int_{G} \theta^{2}\left(\left|a_{1}\right|^{2}|\nabla u|^{2}+a_{2}^{2} u^{2}+f^{2}\right) d x d t \\
\leq & \frac{1}{64} \lambda^{2} \mathbb{E} \int_{\delta}^{T} \int_{G} v^{2} d x d t+3\left|a_{1}\right|_{L_{\mathcal{F}}^{\infty}\left(0, T ; L^{\infty}\left(G ; \mathbb{R}^{n}\right)\right)}^{2} \int_{\delta}^{T} \int_{G}|\nabla v|^{2} d x d t \\
& +3\left|a_{2}\right|_{L_{\mathcal{F}}^{\infty}\left(0, T ; L^{\infty}(G)\right)}^{2} \mathbb{E} \int_{\delta}^{T} \int_{G} v^{2} d x d t+3 \mathbb{E} \int_{\delta}^{T} \int_{G} \theta^{2} f^{2} d x d t .
\end{aligned}
$$


From (2.2)-(2.9), we find

$$
\begin{aligned}
& \frac{1}{4} \lambda \mathbb{E} \int_{\delta}^{T} \int_{G}|\nabla v|^{2} d x d t-C\left(\left|a_{1}\right|_{L_{\mathcal{F}}^{\infty}\left(0, T ; L^{\infty}\left(G ; \mathbb{R}^{n}\right)\right)}^{2}+\left|a_{3}\right|_{L_{\mathcal{F}}^{\infty}\left(0, T ; W^{1, \infty}(G)\right)}^{2}+1\right) \mathbb{E} \int_{\delta}^{T} \int_{G}|\nabla v|^{2} d x d t \\
& -C\left(\left|a_{2}\right|_{L_{\mathcal{F}}^{\infty}\left(0, T ; L^{\infty}(G)\right)}^{2}+\left|a_{3}\right|_{L_{\mathcal{F}}^{\infty}\left(0, T ; W^{1, \infty}(G)\right)}^{2}+1\right) \mathbb{E} \int_{\delta}^{T} \int_{G}|\nabla v|^{2} d x d t \\
& +\left(\frac{1}{8} s \lambda^{2}-\frac{1}{64} \lambda^{2}\right) \mathbb{E} \int_{\delta}^{T} \int_{G} \varphi v^{2} d x d t \\
& \leq C \mathbb{E}\left[|\nabla v(T)|_{L^{2}(G)}^{2}+|\nabla v(\delta)|_{L^{2}(G)}^{2}+s \lambda \varphi(T)|v(T)|_{L^{2}(G)}^{2}+s \lambda \varphi(\delta)|v(\delta)|_{L^{2}(G)}^{2}\right. \\
& \left.\quad+s \lambda \int_{\delta}^{T} \int_{G}(1+\varphi) \theta^{2}\left(f^{2}+g^{2}+|\nabla g|^{2}\right) d x d t\right] .
\end{aligned}
$$

Recalling that

$$
r_{1}=\left|a_{1}\right|_{L_{\mathcal{F}}^{\infty}\left(0, T ; L^{\infty}\left(G ; \mathbb{R}^{n}\right)\right)}^{2}+\left|a_{2}\right|_{L_{\mathcal{F}}^{\infty}\left(0, T ; L^{\infty}(G)\right)}^{2}+\left|a_{3}\right|_{L_{\mathcal{F}}^{\infty}\left(0, T ; W^{1, \infty}(G)\right)}^{2}+1
$$

from inequality (2.10), we know that there exists a $\lambda_{1} \geq \max \left\{C r_{1}, \lambda_{0}\right\}$ such that for all $\lambda \geq \lambda_{1}$, there exists a $s_{0}\left(\lambda_{1}\right)>0$ so that for all $s \geq s_{0}\left(\lambda_{1}\right)$, it holds that

$$
\begin{aligned}
& \lambda \mathbb{E} \int_{\delta}^{T} \int_{G}|\nabla v|^{2} d x d t+s \lambda^{2} \mathbb{E} \int_{\delta}^{T} \int_{G} \varphi v^{2} d x d t \\
\leq & C \mathbb{E}\left[|\nabla v(T)|_{L^{2}(G)}^{2}+|\nabla v(\delta)|_{L^{2}(G)}^{2}+s \lambda \varphi(T)|v(T)|_{L^{2}(G)}^{2}+s \lambda \varphi(\delta)|v(\delta)|_{L^{2}(G)}^{2}\right. \\
& \left.+s \lambda \int_{\delta}^{T} \int_{G}(1+\varphi) \theta^{2}\left(f^{2}+g^{2}+|\nabla g|^{2}\right) d x d t\right],
\end{aligned}
$$

which implies inequality (1.11) immediately.

\section{Proof for Theorem $\mathbf{1 . 2}$}

This section is devoted to the proof of Theorem 1.2. We borrow some ideas from [30].

Proof of Theorem 1.2: Choose $t_{1}$ and $t_{2}$ such that $0<t_{1}<t_{2}<t_{0}$. Set $\alpha_{k}=e^{\lambda t_{k}}$ $(k=0,1,2)$. Let $\rho \in C^{\infty}(\mathbb{R})$ such that $0 \leq \rho \leq 1$ and that

$$
\rho= \begin{cases}1, & t \geq t_{2} \\ 0, & t \leq t_{1} .\end{cases}
$$

Let $z=\rho y$, by means of $y$ solves equation (1.12), we know that $z$ solves

$$
\begin{cases}d z-\sum_{i, j=1}^{n}\left(b^{i j} z_{x_{i}}\right)_{x_{j}} d t=\left[\left(a_{1}, \nabla z\right)+a_{2} z+\rho_{t}(t) y\right] d t+a_{3} z d B(t) & \text { in } Q \\ z=0 & \text { on } \Sigma \\ z(0)=0 & \text { in } G .\end{cases}
$$


Applying Theorem 1.1 with $\psi=t$ and $\delta=0$ to equation (3.2), for $\lambda \geq \lambda_{1}$ and $s \geq s_{0}\left(\lambda_{1}\right)$, we have

$$
\begin{aligned}
& \lambda \mathbb{E} \int_{Q} \theta^{2}|\nabla z|^{2} d x d t+s \lambda^{2} \mathbb{E} \int_{Q} \theta^{2} \varphi|z|^{2} d x d t \\
\leq & C \mathbb{E}\left[\theta^{2}(T)|\nabla z(T)|_{L^{2}(G)}^{2}+s \lambda \varphi(T) \theta^{2}(T)|z(T)|_{L^{2}(G)}^{2}+\int_{Q} \theta^{2}\left|\rho_{t}(t) y\right|^{2} d x d t\right] .
\end{aligned}
$$

From the choice of $\rho$, we see that

$$
\mathbb{E} \int_{Q} \theta^{2}\left|\rho_{t}(t)\right|^{2} y^{2} d x d t \leq C \int_{t_{1}}^{t_{2}} \int_{G} \theta^{2} y^{2} d x d t \leq C \theta^{2}\left(t_{1}\right)|y|_{L_{\mathcal{F}}^{2}\left(0, T ; L^{2}(G)\right)}^{2} .
$$

This, together with inequality (3.3), implies that

$$
\begin{aligned}
& \lambda \theta^{2}\left(t_{0}\right) \mathbb{E} \int_{t_{0}}^{T} \int_{G}|\nabla y|^{2} d x d t+s \lambda^{2} \theta^{2}\left(t_{0}\right) \mathbb{E} \int_{t_{0}}^{T} \int_{G} \varphi|y|^{2} d x d t \\
\leq & \lambda \mathbb{E} \int_{Q} \theta^{2}|\nabla z|^{2} d x d t+s \lambda^{2} \mathbb{E} \int_{Q} \theta^{2} \varphi|z|^{2} d x d t \\
\leq & C \theta^{2}\left(t_{1}\right)|y|_{L_{\mathcal{F}}^{2}\left(0, T ; L^{2}(G)\right)}^{2}+C \mathbb{E}\left(\theta^{2}(T)|\nabla y(T)|_{L^{2}(G)}^{2}+s \lambda \varphi(T) \theta^{2}(T)|y(T)|_{L^{2}(G)}^{2}\right) .
\end{aligned}
$$

Here we utilize the fact that $\theta(t) \leq \theta(s)$ for $t \leq s$.

From inequality (3.5), we see

$$
\begin{aligned}
& \lambda \mathbb{E} \int_{t_{0}}^{T} \int_{G}|\nabla y|^{2} d x d t+s \lambda^{2} \mathbb{E} \int_{t_{0}}^{T} \int_{G} \varphi|y|^{2} d x d t \\
\leq & C \theta^{2}\left(t_{1}\right) \theta^{-2}\left(t_{0}\right)|y|_{L_{\mathcal{F}}^{2}\left(0, T ; L^{2}(G)\right)}^{2}+C \mathbb{E}\left(\theta^{2}(T)|\nabla y(T)|_{L^{2}(G)}^{2}+s \lambda \varphi(T) \theta^{2}(T)|y(T)|_{L^{2}(G)}^{2}\right) .
\end{aligned}
$$

By means of $d\left(y^{2}\right)=2 y d y+(d y)^{2}$, we obtain that

$$
\begin{aligned}
& \mathbb{E} \int_{G}\left|y\left(t_{0}\right)\right|^{2} d x \\
= & \mathbb{E} \int_{G}|y(T)|^{2} d x-\mathbb{E} \int_{t_{0}}^{T} \int_{G}\left[2 y d y+(d y)^{2}\right] d x \\
= & \mathbb{E} \int_{G}|y(T)|^{2} d x-\mathbb{E} \int_{t_{0}}^{T} \int_{G}\left\{2 y\left[\sum_{i, j=1}^{n}\left(b^{i j} y_{x_{i}}\right)_{x_{j}}+\left(a_{1}, \nabla y\right)+a_{2} y\right]+\left(a_{3} y\right)^{2}\right\} d x d t \\
\leq & \mathbb{E} \int_{G}|y(T)|^{2} d x+C \mathbb{E} \int_{t_{0}}^{T} \int_{G}|\nabla y|^{2} d x d t \\
& +\left(\left|a_{1}\right|_{L_{\mathcal{F}}^{\infty}\left(0, T ; L^{\infty}\left(G ; \mathbb{R}^{n}\right)\right)}^{2}+\left|a_{2}\right|_{L_{\mathcal{F}}^{\infty}\left(0, T ; L^{\infty}(G)\right)}+\left|a_{2}\right|_{L_{\mathcal{F}}^{\infty}\left(0, T ; L^{\infty}(G)\right)}^{2} \mathbb{E} \int_{t_{0}}^{T} \int_{G} y^{2} d x d t\right. \\
\leq & \mathbb{E} \int_{G}|y(T)|^{2} d x+C \mathbb{E} \int_{t_{0}}^{T} \int_{G}|\nabla y|^{2} d x d t+C r_{1} \mathbb{E} \int_{t_{0}}^{T} \int_{G} y^{2} d x d t,
\end{aligned}
$$


Recalling $\varphi \geq 1$, from inequality (3.7), we know that there exists a $\lambda_{2}>0$ such that for all $\lambda \geq \lambda_{2}$, it holds that

$$
\begin{aligned}
& \mathbb{E} \int_{G}\left|y\left(t_{0}\right)\right|^{2} d x \\
\leq & \mathbb{E} \int_{G}|y(T)|^{2} d x+C\left(\lambda \mathbb{E} \int_{t_{0}}^{T} \int_{G}|\nabla y|^{2} d x d t+s \lambda^{2} \mathbb{E} \int_{t_{0}}^{T} \int_{G} \varphi y^{2} d x d t\right) .
\end{aligned}
$$

Combing inequality (3.6) and inequality (3.8), for any $\lambda \geq \max \left\{\lambda_{1}, \lambda_{2}\right\}$ and $s \geq s_{0}\left(\lambda_{1}\right)$, we have

$$
\begin{aligned}
& \mathbb{E} \int_{G}\left|y\left(t_{0}\right)\right|^{2} d x \\
\leq & C \theta^{2}\left(t_{1}\right) \theta^{-2}\left(t_{0}\right)|y|_{L_{\mathcal{F}}^{2}\left(0, T ; L^{2}(G)\right)}^{2}+C \mathbb{E}\left(\theta^{2}(T)|\nabla y(T)|_{L^{2}(G)}^{2}+s \lambda \varphi(T) \theta^{2}(T)|y(T)|_{L^{2}(G)}^{2}\right) .
\end{aligned}
$$

Now we fix $\lambda_{3}=\max \left\{\lambda_{1}, \lambda_{2}\right\}$, from inequality (3.9), we get

$$
\mathbb{E} \int_{G}\left|y\left(t_{0}\right)\right|^{2} d x \leq C \theta^{2}\left(t_{1}\right) \theta^{-2}\left(t_{0}\right)|y|_{L_{\mathcal{F}}^{2}\left(0, T ; L^{2}(G)\right)}^{2}+C \theta^{2}(T) \mathbb{E}|y(T)|_{H^{1}(G)}^{2} .
$$

Replacing $C$ by $C e^{s_{0} e^{\lambda_{3} T}}$, from inequality (3.10), for any $s>0$, it holds that

$$
\mathbb{E} \int_{G}\left|y\left(t_{0}\right)\right|^{2} d x \leq C e^{-2 s\left(e^{\lambda_{3} t_{1}}-e^{\lambda_{3} t_{0}}\right)}|y|_{L_{\mathcal{F}}^{2}\left(0, T ; L^{2}(G)\right)}^{2}+C e^{C s} \mathbb{E}|y(T)|_{H^{1}(G)}^{2} .
$$

Choosing $s \geq 0$ which minimize the right-hand side of inequality (3.11), we obtain that

$$
\mathbb{E}\left|y\left(t_{0}\right)\right|_{L^{2}(G)}^{2} \leq C|y|_{L_{\mathcal{F}}^{2}\left(0, T ; L^{2}(G)\right)}^{1-\theta} \mathbb{E}|y(T)|_{H^{1}(G)}^{\theta},
$$

with

$$
\theta=\frac{2\left(e^{\lambda_{3} t_{0}}-e^{\lambda_{3} t_{1}}\right)}{C+2\left(e^{\lambda_{3} t_{0}}-e^{\lambda_{3} t_{1}}\right)}
$$

\section{Proof of Theorem 1.4}

Thie section is devoted to proving Theorem 1.4. We borrow some ideas in [30] again .

Proof of Theorem 1.4: From the assumptions on $b_{1}, b_{2}, b_{3}, R$ and $h$, and by Lemma 1.2, we know equation (1.16) admits a unique strong solution. For arbitrary small $\varepsilon>0$, we choose $t_{1}$ and $t_{2}$ such that

$$
0<t_{0}-\varepsilon<t_{1}<t_{2}<t_{0}
$$

Let $\chi \in C^{\infty}(\mathbb{R})$ be a cut-off function such that $0 \leq \chi \leq 1$ and that

$$
\chi= \begin{cases}1, & t \leq t_{1} \\ 0, & t \geq t_{2}\end{cases}
$$


Put $y=R z$ (recall $(1.17)$ for $R$ ) in $\left[0, t_{2}\right] \times G$. Since $y$ is a strong solution of equation (1.16), we know that $z$ solves

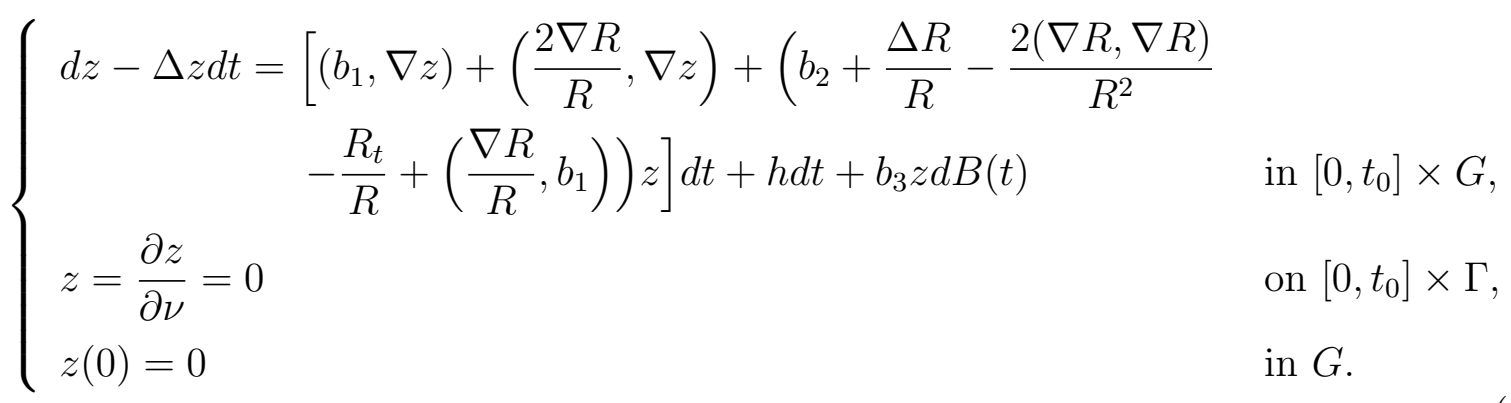

Setting $u=z_{x_{1}}$, noting $z$ is the strong solution of equation (4.2) and $z_{x_{1}}=\frac{\partial z}{\partial \nu}=0$ on $\left(\{0\} \times G^{\prime}\right) \cup\left(\{l\} \times G^{\prime}\right)$, we know that $u$ is the weak solution of the following equation:

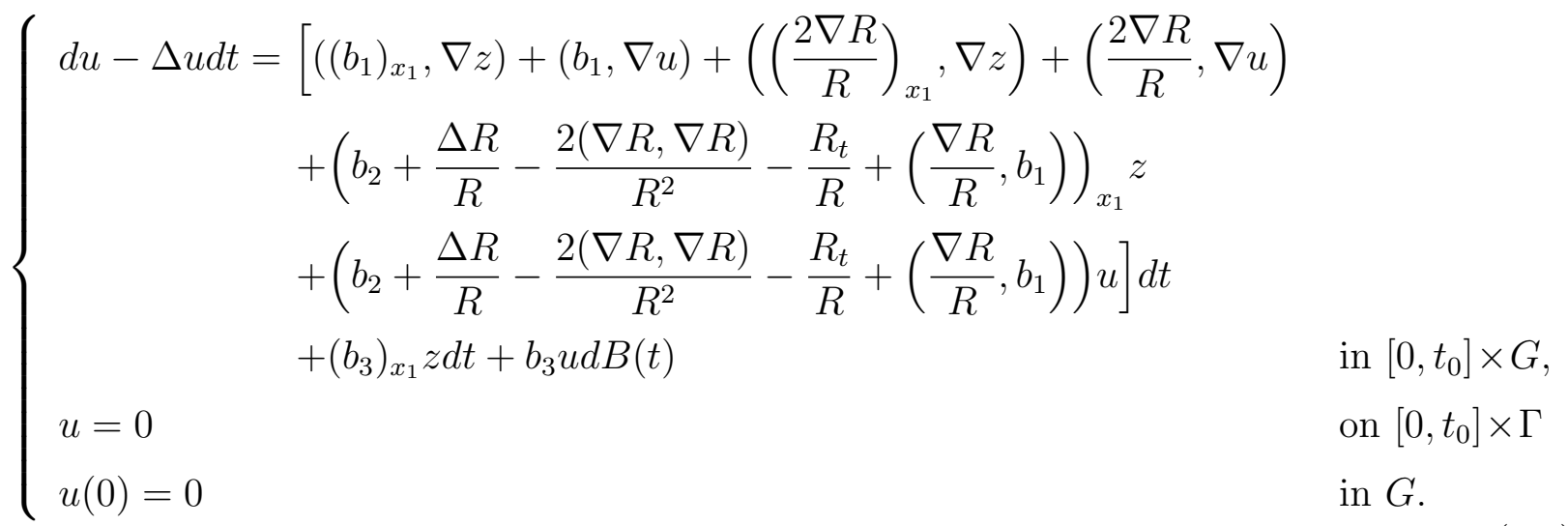

Set $w=\chi u$. Then we know that $w$ is a weak solution of the following equation:

$$
\left\{\begin{array}{rlrl}
d w-\Delta w d t= & {\left[\left(\left(b_{1}\right)_{x_{1}}, \chi \nabla z\right)+\left(b_{1}, \nabla w\right)+\left(\left(\frac{2 \nabla R}{R}\right)_{x_{1}}, \chi \nabla z\right)+\left(\frac{2 \nabla R}{R}, \nabla w\right)\right.} & \\
& +\left(b_{2}+\frac{\Delta R}{R}-\frac{2(\nabla R, \nabla R)}{R^{2}}-\frac{R_{t}}{R}+\left(\frac{\nabla R}{R}, b_{1}\right)\right)_{x_{1}} \chi z & \\
& \left.+\left(b_{2}+\frac{\Delta R}{R}-\frac{2(\nabla R, \nabla R)}{R^{2}}-\frac{R_{t}}{R}+\left(\frac{\nabla R}{R}, b_{1}\right)\right) w\right] d t & & \text { in }\left[0, t_{0}\right] \times G, \\
& +\left(b_{3}\right)_{x_{1}} \chi z d B(t)+b_{3} w d B(t)-\chi^{\prime} u d t & \text { on }\left[0, t_{0}\right] \times \Gamma, \\
w=0 & & \text { in } G .
\end{array}\right.
$$

By means of $u=z_{x_{i}}$ and $z\left(t, 0, x^{\prime}\right)=y\left(t, 0, x^{\prime}\right)=0$ for $\left(t, x^{\prime}\right) \in\left(0, t_{0}\right) \times G^{\prime}$, we see

$$
\chi z=\chi \int_{0}^{x_{1}} u\left(t, \eta, x^{\prime}\right) d \eta=\int_{0}^{x_{1}} w\left(t, \eta, x^{\prime}\right) d \eta .
$$

This, together with equation (4.4), implies that $w$ is the weak solution of the following 
equation:

$$
\left\{\begin{aligned}
d w-\Delta w d t= & {\left[\left(b_{1}, \nabla w\right)+\left(\frac{2 \nabla R}{R}, \nabla w\right)+\left(\left(b_{1}\right)_{x_{1}}, \nabla \int_{0}^{x_{1}} w\left(t, \eta, x^{\prime}\right) d \eta\right)\right.} & \\
& +\left(\left(\frac{2 \nabla R}{R}\right)_{x_{1}}, \nabla \int_{0}^{x_{1}} w\left(t, \eta, x^{\prime}\right) d \eta\right) & \\
& +\left(b_{2}+\frac{\Delta R}{R}-\frac{2(\nabla R, \nabla R)}{R^{2}}-\frac{R_{t}}{R}+\left(\frac{\nabla R}{R}, b_{1}\right)\right) w & \\
& \left.+\left(b_{2}+\frac{\Delta R}{R}-\frac{R_{t}}{R}+\left(\frac{\nabla R}{R}, b_{1}\right)\right)_{x_{1}} \int_{0}^{x_{1}} w\left(t, \eta, x^{\prime}\right) d \eta\right] d t & \\
& +\left(b_{3}\right)_{x_{1}} \chi \int_{0}^{x_{1}} u\left(t, \eta, x^{\prime}\right) d \eta d B(t)+b_{3} w d B(t)-\chi^{\prime} u d t & \text { in }\left[0, t_{0}\right] \times G, \\
w=0 & & \text { on }\left[0, t_{0}\right] \times \Gamma, \\
w=0 & & \text { in } G .
\end{aligned}\right.
$$

Applying Theorem 1.1 to equation (4.6) with $\psi(t)=-t$, noting that $w(0)=0$, and that $w\left(t_{0}\right)=\chi\left(t_{0}\right) u\left(t_{0}\right)=0$, we get

$$
\begin{aligned}
& \mathbb{E} \int_{0}^{t_{0}} \int_{G} \theta^{2}\left(\lambda|\nabla w|^{2}+s \lambda^{2} w^{2}\right) d x d t \\
\leq & C \mathbb{E} \int_{0}^{t_{0}} \int_{G} \theta^{2}\left|\chi^{\prime} u\right|^{2} d x d t \\
& +C r_{1} \mathbb{E} \int_{0}^{t_{0}} \int_{G} \theta^{2}\left(\left|\int_{0}^{x_{1}} w\left(t, \eta, x^{\prime}\right) d \eta\right|^{2}+\left.\left|\int_{0}^{x_{1}}\right| \nabla w\left(t, \eta, x^{\prime}\right) d \eta\right|^{2}\right) d x d t .
\end{aligned}
$$

Since

we know

$$
\left|\int_{0}^{x_{1}} w\left(t, \eta, x^{\prime}\right) d \eta\right|^{2} \leq l \int_{0}^{l}\left|w\left(t, \eta, x^{\prime}\right)\right|^{2} d \eta
$$

$$
\begin{aligned}
\int_{0}^{t_{0}} \int_{G} \theta^{2}\left|\int_{0}^{x_{1}} w\left(t, \eta, x^{\prime}\right) d \eta\right|^{2} d x d t & \leq l \int_{0}^{l} d x_{1} \int_{0}^{t_{0}} \int_{G^{\prime}} \int_{0}^{l} \theta^{2}\left|w\left(t, \eta, x^{\prime}\right)\right|^{2} d \eta d x^{\prime} d t \\
& \leq l^{2} \int_{0}^{t_{0}} \int_{G} \theta^{2}\left|w\left(t, \eta, x^{\prime}\right)\right|^{2} d \eta d x^{\prime} d t
\end{aligned}
$$

By virtue of

$$
\nabla \int_{0}^{x_{1}} w\left(t, \eta, x^{\prime}\right) d \eta=\int_{0}^{x_{1}} \nabla w\left(t, \eta, x^{\prime}\right) d \eta+w\left(t, 0, x^{\prime}\right)=\int_{0}^{x_{1}} \nabla w\left(t, \eta, x^{\prime}\right) d \eta
$$

we get that

$$
\begin{aligned}
\int_{0}^{t_{0}} \int_{G} \theta^{2}\left|\nabla \int_{0}^{x_{1}} w\left(t, \eta, x^{\prime}\right) d \eta\right|^{2} d x d t & =\int_{0}^{t_{0}} \int_{G} \theta^{2}\left|\nabla \int_{0}^{x_{1}} w\left(t, \eta, x^{\prime}\right) d \eta\right|^{2} d x d t \\
& \leq l \int_{0}^{l} d x_{1} \int_{0}^{t_{0}} \int_{G^{\prime}} \int_{0}^{l} \theta^{2}\left|\nabla w\left(t, \eta, x^{\prime}\right)\right|^{2} d \eta d x^{\prime} d t \\
& \leq l^{2} \int_{0}^{t_{0}} \int_{G} \theta^{2}\left|\nabla w\left(t, \eta, x^{\prime}\right)\right|^{2} d \eta d x^{\prime} d t .
\end{aligned}
$$


From inequality $(4.7)-(4.9)$, we obtain that

$$
\begin{aligned}
& \mathbb{E} \int_{0}^{t_{0}} \int_{G} \theta^{2}\left(\lambda|\nabla w|^{2}+s \lambda^{2} w^{2}\right) d x d t \\
\leq & C \mathbb{E} \int_{0}^{t_{0}} \int_{G} \theta^{2}\left|\chi^{\prime} u\right|^{2} d x d t+C l^{2} r_{1} \mathbb{E} \int_{0}^{t_{0}} \int_{G} \theta^{2}\left(|\nabla w|^{2}+|w|^{2}\right) d x d t .
\end{aligned}
$$

Thus, we know that there is a $\lambda_{4}=\max \left\{C r_{1}, \lambda_{1}\right\}$ such that for all $\lambda \geq \lambda_{4}$, there exists an $s_{1}\left(\lambda_{4}\right)>0$ so that for all $s \geq s_{1}\left(\lambda_{4}\right)$, it holds that

$$
\mathbb{E} \int_{0}^{t_{0}} \int_{G} \theta^{2}\left(\lambda|\nabla w|^{2}+s \lambda^{2} w^{2}\right) d x d t \leq C \mathbb{E} \int_{0}^{t_{0}} \int_{G} \theta^{2}\left|\chi^{\prime} u\right|^{2} d x d t
$$

Fix $\lambda=\lambda_{4}$, by the property of $\chi($ see $(4.1))$, we find

$$
\mathbb{E} \int_{0}^{t_{0}} \int_{G} \theta^{2}\left|\chi^{\prime} u\right|^{2} d x d t \leq e^{2 s e^{-\lambda_{4} t_{1}}} \mathbb{E} \int_{Q}|u|^{2} d x d t \leq e^{2 s e^{-\lambda_{4} t_{1}}}\left|y_{x_{1}}\right|_{L_{\mathcal{F}}^{2}\left(0, T ; L^{2}(G)\right)}^{2}
$$

This, together with inequality (4.11), implies that for all $s \geq s_{1}$, it holds that

$$
\begin{aligned}
e^{2 s e^{-\lambda_{4}\left(t_{0}-\varepsilon\right)} \mathbb{E} \int_{0}^{t_{0}-\varepsilon} \int_{G}\left(|\nabla w|^{2}+s w^{2}\right) d x d t} & \leq \mathbb{E} \int_{0}^{t_{0}-\varepsilon} \int_{G} \theta^{2}\left(|\nabla w|^{2}+s w^{2}\right) d x d t \\
& \leq \mathbb{E} \int_{0}^{t_{0}} \int_{G} \theta^{2}\left(|\nabla w|^{2}+s w^{2}\right) d x d t \\
& \leq C e^{2 s e^{-\lambda_{4} t_{1}}}\left|y_{x_{1}}\right|_{L_{\mathcal{F}}^{2}\left(0, T ; L^{2}(G)\right) .}^{2}
\end{aligned}
$$

From inequality (4.13), we have

$$
|w|_{L_{\mathcal{F}}^{2}\left(0, T ; H^{1}(G)\right)}^{2} \leq C e^{2 s\left(e^{-\lambda_{4} t_{1}}-e^{-\lambda_{4}\left(t_{0}-\varepsilon\right)}\right)}\left|y_{x_{1}}\right|_{L_{\mathcal{F}}^{2}\left(0, T ; L^{2}(G)\right)}^{2} .
$$

Recalling that $t_{0}-\varepsilon<t_{1}$, we know $e^{-\lambda_{4} t_{1}}-e^{-\lambda_{4}\left(t_{0}-\varepsilon\right)}<0$. Letting $s \rightarrow+\infty$, we obtain that

$$
w=0 \text { in }\left(0, t_{0}-\varepsilon\right) \times G, P \text {-a.s. }
$$

This, together with equality (4.5), implies that

$$
z=0 \text { in }\left(0, t_{0}-\varepsilon\right) \times G, \quad P \text {-a.s. }
$$

which means

$$
h=0 \text { in }\left(0, t_{0}-\varepsilon\right) \times G^{\prime}, P \text {-a.s. }
$$

Since $\varepsilon>0$ is arbitrary, the proof of Theorem 1.4 is completed.

\section{Acknowledgments.}

The author would like to thank the anonymous referees for helpful comments. 


\section{References}

[1] W. F. Ames and B. Straughan, Non-Standard and Improperly Posed Problems, Academic Press, San Diego, 1997.

[2] V. Barbu, A. Răscanu and G. Tessitore, Carleman estimate and controllability of linear stochastic heat equatons, Appl. Math. Optim., 47(2003), 97-120.

[3] G. Bao, S.-N. Chow, P. Li and H. Zhou, Numerical solution of an inverse medium scattering problem with a stochastic source, Inverse Problems, 26 (2010), 074014 (23pp).

[4] A. L. Bukhgeim and M. V. Klibanov, Global uniqueness of class of multidimentional inverse problems, Soviet Mathematics Doklady, 24(1981), 244-247.

[5] Z. Brzezniak and M. Neklyudov, Backward uniqueness and the existence of the spectral limit for some parabolic SPDEs, http://arxiv.org/abs/0806.0616.

[6] A. P. Calderón, Uniqueness in the Cauchy problem for partial differential equations, Ann. of Math., 80(1958), 16-36.

[7] J. R. Cannon and S. Pérez Esteva, Uniqueness and stability of $3 D$ heat sources, Inverse Problems, 7(1991), 57-62.

[8] T. Carleman, Sur un problème d'unicité pour les systèmes d'équations aux dérivées partielles à deux variables réelles, Mat. Astr. Fysik., 26B(1939), 1-9.

[9] L. Cavalier and A. Tsybakov, Sharp adaptation for inverse problems with random noise, Probab. Theory Relat. Fields, 123 (2002), 323-354.

[10] M. Choulli and M. Yamamoto, Conditional stability in determining a heat source, J. Inverse Ill-Posed Probl., 12(2004), 233-243.

[11] G. Da Prato and J. Zabczyk, Stochastic Equations in Infinite Dimensions, Cambridge University Press, Cambridge, 1992.

[12] L. Escauriaza, G. Seregin and V. Šverák, Backward uniqueness for parabolic equations, Arch. Ration. Mech. Anal., 169(2003), 147-157.

[13] A. V. Fursikov and O. Yu. Imanuvilov, Controllability of Evolution Equations, Lectures Notes Series, vol. 34, Seoul National University, Seoul, 1996.

[14] J. M. Ghidaglia, Some Backward Uniqueness Results, Nonlinear Analysis. Theory, Methods and Applications, 10 (1986), 777-790.

[15] L. Hörmander, The Analysis of Linear Partial Differential Operators, vol III, Springer-Verlag, 1985.

[16] I.A. Ibragimov and R.Z. Khas'minskii, Estimation problems for coefficients of stochastic partial differential equations. Part I, Theory Probab. Appl., 43 (1999), 370-387.

[17] O. Yu. Imanuvilov and M. Yamamoto, Lipschitz stability in inverse parabolic problems by the Carleman estimate, Inverse Problems, 14(1998), 1229-1245.

[18] V. Isakov, Inverse Problems for Partial Differential Equations, Springer, New York, 2006.

[19] B. T. Johansson and D. Lesnic, A variational method for identifying a spacewise dependent heat source, IMA J. Appl. Math., 72(2007), 748-760.

[20] B. T. Johansson and M. Pricop, A method for identifying a spacewise dependent heat source under stochastic noise interference, Inverse probl. Sci. En., 18(2010), 51-63.

[21] M. V. Klibanov, Inverse problems and Carleman estimates, Inverse Problems, 8(1992), 575-596.

[22] M. V. Klibanov and A. Timonov, Carleman Estimates for Coefficient Inverse Problems and Numerical Applications, Inverse and Ill-Posed Problems Series, VSP, Utrecht, 2004. 
[23] I. Kukavica, Backward uniqueness for solutions of linear parabolic equations, P. Am. Math. Soc., 132(2004), 1755-1760.

[24] M. Lees and M. H. Protter, Unique continuation for parabolic differential equations and inequalities, Duke Math. J., 28(1961), 369-382.

[25] E. Pardoux, Équations aux dérivées partielles stochastiques non linéaires monotones, $\mathrm{Ph} \mathrm{D}$ Thesis, Université Paris XI, 1975.

[26] K.-D. Phung and G. Wang, Quantitative unique continuation for the semilinear heat equation in a convex domain, J. Funct. Anal., 259(2010), 1230-1247.

[27] D. D. Santo and M. Prizzi, Backward uniqueness for parabolic operators whose coefficients are nonLipschitz continuous in time, J. Math. Pure. Appl., 84(2005), 471-491.

[28] S. Tang and X. Zhang, Null controllability for forward and backward stochastic parabolic equations, SIAM J. Control Optim., 48(2009), 2191-2216.

[29] M. Yamamoto, Uniqueness and stability in multidimensional hyperbolic inverse problems, J. Math. Pures. Appl., 78(1999), 65-98.

[30] M. Yamamoto, Carleman estimates for parabolic equations and applications, Inverse Problems, 25(2009), 123013. 